\title{
Research on 3 Dimensional Visualization of Geological Environment Information
}

\author{
Tan Dunming \\ The First Institute of PLAAF \\ Beihang University \\ Beijing, China \\ Bai Peng \\ Chengdu Institute of Survey\&Investigation \\ Chengdu, China
}

\author{
Xue Qunwei \\ China Institute of Geological Environment Monitoring
}

\begin{abstract}
Traditional 2 dimensional visualization method of geological data is carried out in 3 dimensional by OpenGL. This include process and visualize of large scale data of digital elevation model height data, remote sensing texture data, geological environment information data of point, line and polygon types together with geological body data. The result proves that 2 dimensional visualization methods of geological data can be replaced by 3 dimensional with better intuitive and effective, which is also a developing trend in the future.
\end{abstract}

Keywords-Large terrain, LOD, Geological environment information

\section{INTRODUCTION}

All manuscripts must be in English. These guidelines include complete descriptions of the fonts, spacing, and related information for producing your proceedings manuscripts. Please follow them and if you have any questions, direct them to the production editor in charge of your proceedings at Conference Publishing Services (CPS): Phone +1 (714) 821-8380 or Fax +1 (714) 761-1784.

Geological data is a core component of Geographic Information System(GIS). Compared with traditional geological data systems, which use maps drawn by hand, computer aided GIS, especially in 3 dimensional, make a revolution in geological environment information visualization method.

Currently, most of the 3 dimensional GIS visualization research focus on how to combing large scale digital elevation model height data and remote sensing texture data. "Virtual GIS" was designed for height fields presented at Siggraph '96 [1]. It provides the means for visualizing terrain models consisting of elevation and imagery data, along with GIS raster layers, protruding features, buildings, vehicles and other objects.

Continuous LOD (Level of Detail) was studied for realtime rendering of large scale height fields and imagery data. It uses a compact and efficient regular grid representation, and employs a variable screen-space threshold to bind the maximum error of the projected image. Appropriate level of detail is computed and generated dynamically in real-time, allowing for smooth changes of resolution across areas of the surface [2]. However, this algorithm still has a major disadvantage. With the viewpoint moving, the triangulation is continuously changing, resulting in a phenomenon called vertex popping. Stefan introduced a new, rapid geomorphing algorithm of real-time generation of continuous LOD for height fields to eliminate these artifacts, which operates top down on a quad tree data structure [3].

Incremental triangle stripping and priority computation deferral lists were introduced in "ROAM" to optimize rendering performance [4]. The execution time is proportionate to the number of triangle changes per frame, which is typically a few percent of the output mesh size. However, the imagery data is still limited because of hardware architecture. The clipmap, which is a virtual mipmap, use a dynamic texture representation that efficiently caches textures of arbitrarily large size in a finite amount of physical memory for rendering at real-time rates $[5]$.

Though there are several exploration of 3 dimensional geological data visualization[6][7][8][9], most of the geological systems are based on 2 dimensional currently, which limits the information shown to users. Meanwhile, they mostly focus on visualization of large scale terrain dataset of height fields and imagery data.

In this paper, through a practical case study, based on 3 dimensional visualization of large scale height fields and imagery data, visualization of geological environment information data and geological body data is explained in detail. The principles and methods are not only applicable to geological, but also to any other GIS application.

\section{PREPRocess Of Height FiEldS AND IMAGERy DATA}

3 dimensional visualization of height fields and imagery data is the basis of GIS, this is also a prerequisites. Both the height fields and imagery data need some preprocess.

An convenient way to get height fields and imagery data is to download form the internet. For example, height fields data can be obtained from NASA SRTM(Shuttle Radar Topography Mission) project, which provide on earth height fields with resolution of 30 by 30 meters. Besides, google earth provide calibrated satellite imagery data, and can be download by some software tools. 
Height fields data is regular grids in $\mathrm{x}$ and $\mathrm{y}$ direction with height in $\mathrm{z}$ direction. Most of current terrain rendering algorithms require the height fields data to be a grids of $2^{n}+1$, so the first step is to rescale the size of the given height fields data. There are many scaling methods, include box scaling, bilinear scaling, bspline scaling, bicubic scaling, Catmull-Rom scaling and Lanczos scaling. Of all the scaling methods, bspline scaling is the most suitable to resample terrain for scaling, because it is a smooth interpolation and brings minimum change to the features of height fields.

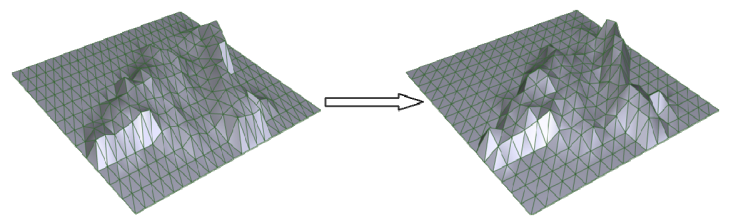

Figure 1. Rescale the height fields data to resolution of $2^{n}+1$

Imagery data is optional. If there is no imagery data, the height fields data can be convert to grayscale image or use user defined or color lookup table to generate imagery data. Imagery data is used as textures of rendering pipeline. Modern graphics card needs the texture size to be $2^{n}$, so imagery data also need to be resampled.

Imagery data with high resolution makes more realistic the geological system and become an developing trend. However, the texture size is restrict by graphics cards at most to $4096 * 4096$ for most graphics cards of personal computer. Thus, in order to use large resolution imagery data, the height fields and imagery data may need to be cut to tiles, this also makes dynamic paging in visible tiles and paging out invisible tiles to promote rendering performance.

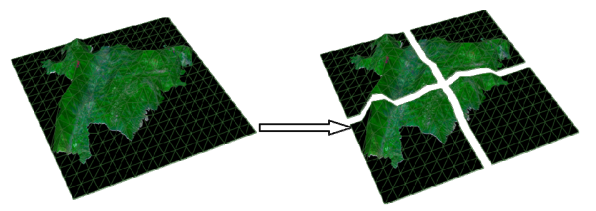

Figure 2. Generate tiled height fields with imagery

In order to prove performance, multi-resolution tiles are used. Shown in Figure 3, each tile have different resolution according to the rendering ability of the target rendering system. The specific resolution level is choose with considering of camera height from the height fields and rendering frames per seconds.

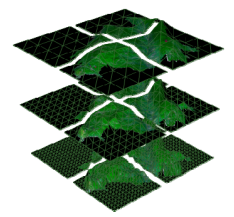

Figure 3. Multi-resolution tiles to promote performance

\section{LOD RENDERING OF HEIGHT FIELDS AND IMAGERY DATA}

LOD rendering algorithms described in [2][3] are used for real-time rendering of height fields with imagery. The LOD rendering algorithm build quadtree structure from the height fields grids. Then it choose appropriate quadtree when rendering. The rendering algorithm is based on two major constrains shown in formula 1 and 2 , where 1 is the distance from viewer position and $d$ is the edge length of quadtree, $d h_{i}$ is the elevation difference at the mid-point of adjacent quadtree node. Constant values $\mathrm{C}$ and $\mathrm{c}$ respect for global and local error constrains, which can be used to choose appropriate rendering accuracy. Shown in Figure 4.

$$
\begin{aligned}
& \frac{l}{d}<C \\
& \frac{1}{d} \max _{i=1 . .5}\left|d h_{i}\right|<c
\end{aligned}
$$
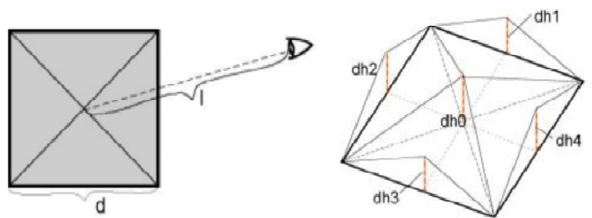

Figure 4. LOD rendering algorithm of quad tree with error constrains

Finally, the rendering system use LOD algorithm to render the generated tiles shown in Figure 3 with imagery. The rendering result with height fields in wireframe is shown in Figure 5.

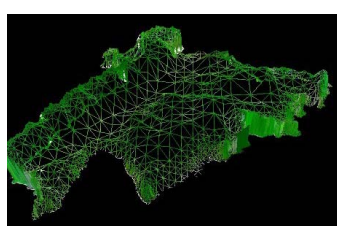

Figure 5. LOD rendering of height fields and imagery

\section{Visualization Of GeOlogical EnVironment INFORMATION DATA}

Geological environment information data refers to point, line and polygon types data such as points of landslide, lines of contours, roads, regions of disaster and administration. These information are traditionally marked in 2 dimensional maps. In 3 dimensional visualization of geological environment information, they are displayed according to their data types.

First, the point data is rendered with icons or text of billboards. For example, landslide can use icons to alarm the user and village names are rendered by their names directly using texts shown in Figure 6. Billboard is a technique that adjusts an object's orientation so that it faces some target, usually the camera. 

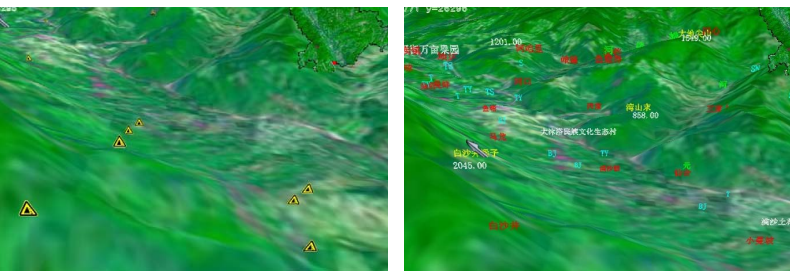

Figure 6. Data of point type are rendered as icons or texts

Second, the line data is rendered as 3 dimensional lines directly. However, the lines are raised in $\mathrm{z}$ direction in order to not be hidden by height fields. The attribute assigned to the line such as position, length, names and so on can be acquired by mouse selection. See Figure 7 for details.

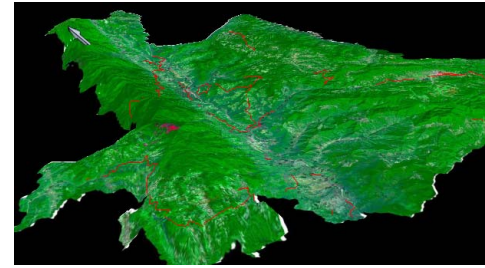

Figure 7. Data of lines type are rendered directly

Third, the polygon data is rendered as textures with remote sensing texture blended with an alpha value. These is accomplished by dynamic blending the polygon data and remote sensing texture data together by Frame Buffer Objects (FBO) of OpenGL at run time. The blended image is used to replace the original imagery data for height fields. Polygon is tesselated by OpenGL utility library of gluTess. Polygon region can be rendered with different colors to represent with different attributes. Similar to the line data, polygon data can also be picked to get specific attribute information shown in Figure 8.
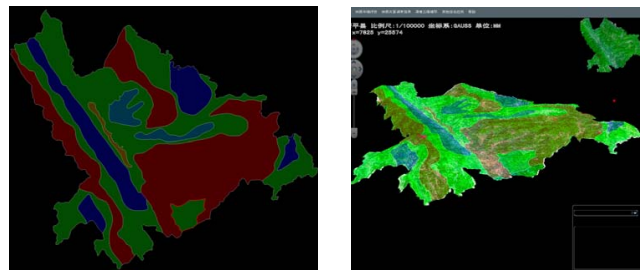

Figure 8. Blended polygon data and 3 dimensional visualization

\section{Visualization Of Geological Body Data}

Geological body data refers to drilling, stratum, section and landslide. These body data are traditionally described by section views and is hard to image spatial position. In 3 dimensional visualization of geological environment information, they are directly display as what they truly exist with special design to respect their attributes and identities.

First, drilling is rendered directly. Unlike 2 dimensional visualization, which need to be rendered by different views by points or lines. They are rendered as cylinders with icons rendering as textures blended with attributes as colors.
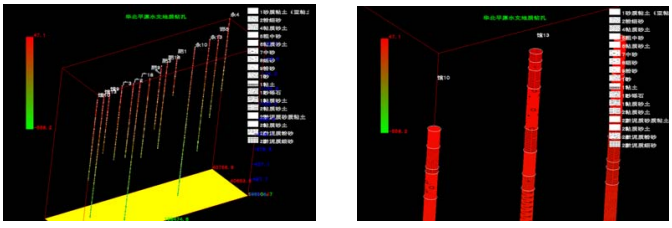

Figure 9. Visualization of drillings with icons as textures

Second, drilling is connected into stratums by user interaction according to their experience with the attributes and depth taking into consideration. The stratums between adjacent drilling can be further connected as sections.
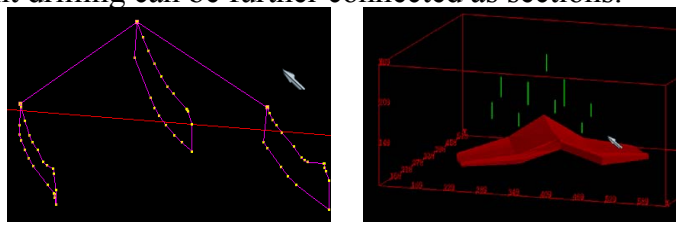

Figure 10. Visualization of stratums and sections marked in green circle
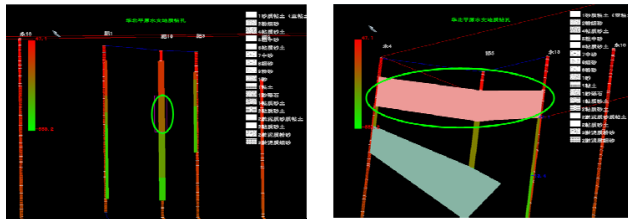

Figure 11. Sections are connected to form a landslide
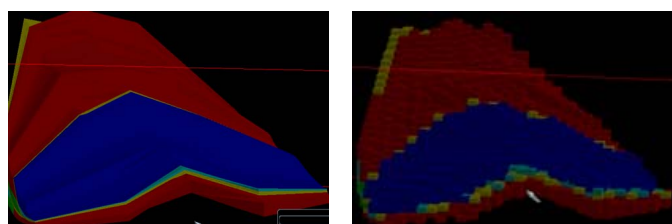

Figure 12. Visualization of landslide with surface and solid mode

Third, by connecting sections with the same attributes and relative position, finally they make the landslide. The landslide can be represented by surface model or interpolated into bodies of cubes of solid model. Shown in Figure 11 and Figure 12.

\section{SUMMARIES}

3 Dimensional visualization of geological data is studied in this paper. The system is based on 3 dimensional visualization of GIS, especially large scale terrain visualization with LOD tiles. Geological environment information data and geological body data are visualized with OpenGL integrated as a whole to the GIS system. The result shows an approach to traditional 2 dimensional GIS system of how to carry out in 3 dimensional.

\section{REFERENCES}

[1] David Koller, Peter Lindstrom, William Ribarsky, Larry F. Hodges, Nick Faust, and Gregory Turner. Virtual GIS: A real time 3D geographic information system. In G. Nielson and D. Silver, editors, Proceedings Visualization '95, pages 94-100. IEEE Computer Society Press, 1996.

[2] Peter Lindstrom, David Koller, William Ribarsky, Larry F. Hodges, Nick Faust, and Gregory Turner. Real-time, continuous level of detail 
rendering of height fields. In Computer Graphics (Proceedings Siggraph '96), pages 109-118, 1996.

[3] Rottger, S. and Heidrich, W. and Slusallek, P. and Seidel, H.P. and others. Real-time generation of continuous levels of detail for height fields. Proc. WSCG'98, pages 315-322. Citeseer, 1998.

[4] Duchaineau, M. and Wolinsky, M. and Sigeti, D.E. and Miller, M.C. and Aldrich, C. and Mineev-Weinstein, M.B. ROAMing terrain: realtime optimally adapting meshes. Proceedings of the 8th Conference on Visualization'97, pages 81-88. IEEE Computer Society Press, 1997.

[5] Tanner, C.C. and Migdal, C.J. and Jones, M.T. The clipmap: a virtual mipmap. Proceedings of the 25th annual conference on Computer graphics and interactive techniques, pages 151-158. ACM, 1998.
[6] Pouliot, J. and Lachance, B. and Brisebois, A. and Rabeau, O. and Kirkwood, D. 3D geological modeling: Are GIS or CAD appropriate. Proceedings of the International Society for Photogrammetry and Remote Sensing Workshop, pages 2-3.2003.

[7] $\mathrm{Wu}, \mathrm{Q}$. and $\mathrm{Xu}, \mathrm{H}$. and Zou, X. An effective method for 3D geological modeling with multi-source data integration. Computers \& Geosciences, pages 35-43. Elsevier, 2005.

[8] Mingtao, W.L.H.C.J. 3D Geological Solid Modeling Technology and Its Application in Engineering. Metal Mine. 2006.

[9] Qingyuan, L. and Zongjian, L. and Chengming, L.The Status and Development of 3DGIS Study. DEVELOPMENTS IN SURVEYING AND MAPPING. 2000. 